\title{
Rapid simultaneous acquisition of macromolecular tissue volume, susceptibility, and relaxometry maps
}

Fang Frank Yu $1{ }^{1,}$, Susie Yi Huang $2,3,4$, Thomas Witzel ${ }^{5}$, Ashwin Kumar6, Congyu Liao $^{7}$, Tanguy Duval ${ }^{8}$, Julien Cohen-Adad ${ }^{8}$, and Berkin Bilgic ${ }^{2,3,4}$

${ }^{1}$ Radiology, University of Texas Southwestern Medical Center, Dallas, TX, United States,

${ }^{2}$ Department of Radiology, Harvard Medical School, Boston, MA, United States

${ }^{3}$ Harvard-MIT Health Sciences and Technology, Massachusetts Institute of Technology, Cambridge, MA, United States

${ }^{4}$ Athinoula A. Martinos Center for Biomedical Imaging, Charlestown, MA, United States, ${ }^{5} \mathrm{Q}$ Bio, Inc., Redwood City, CA, United States,

${ }^{6}$ Vanderbilt University, Nashville, TN, United States,

${ }^{7}$ Radiological Sciences Laboratory, Stanford Medicine, Stanford, CA, United States.

8 Institute of Biomedical Engineering, Ecole Polytechnique de Montreal, Montreal, QC, Canada.

${ }^{*}$ Corresponding author.

Fang Frank Yu, MD

UT Southwestern Medical Center

5323 Harry Hines Blvd

Dallas, TX 75390

Ph: 214-648-7813

Fax: 214-648-3904

frankf.yu@utsouthwestern.edu 
Total word count: 2986

Research reported in this publication was supported by the National Institutes of Health (NIBIB R01 EB028797 [B.B.], U01 EB025162 [B.B.], U01 EB026996 [B.B.], P41 EB030006 [B.B.], NINDS K23 NS096056 [S.Y.H.], NIA R21AG067562 [S.Y.H.]), NVIDIA GPU grant (B.B.), American Society of Neuroradiology Boerger Research Fund in Alzheimer's Disease and Neurocognitive Disorders (S.Y.H.), Marilyn Hilton Award for Innovation in MS Research from the Conrad N. Hilton Foundation (S.Y.H.), MGH Claflin Distinguished Scholar Award (S.Y.H.).

The authors do not have any conflicts of interest to report. 


\section{$\underline{\text { Abstract }}$}

\section{Purpose}

A major obstacle to the clinical implementation of quantitative MR is the lengthy acquisition time required to derive multi-contrast parametric maps. We sought to reduce the acquisition time for quantitative susceptibility mapping (QSM) and macromolecular tissue volume (MTV) by acquiring both contrasts simultaneously by leveraging their redundancies. The Joint Virtual Coil concept with generalized autocalibrating partially parallel acquisitions (JVC-GRAPPA) was applied to reduce acquisition time further.

\section{Methods}

Three adult volunteers were imaged on a 3T scanner using a multi-echo 3D GRE sequence acquired at three head orientations. MTV, QSM, R2* ${ }^{*}$ T1, and proton density maps were reconstructed. The same sequence (GRAPPA $R=4$ ) was performed in subject \#1 with a single head orientation for comparison. Fully sampled data was acquired in subject \#2, from which retrospective undersampling was performed $(R=6$ GRAPPA and R=9 JVC-GRAPPA). Prospective undersampling was performed in subject \#3 (R=6 GRAPPA and R=9 JVC-GRAPPA) using gradient blips to shift k-space sampling in later echoes.

\section{Results}

Subject \#1's multi-orientation and single-orientation MTV maps were not significantly different based on RMSE. For subject \#2, the retrospectively undersampled JVCGRAPPA and GRAPPA generated similar results as fully sampled data. This approach was validated with the prospectively undersampled images in subject \#3. Using QSM, $\mathrm{R} 2^{*}$, and MTV, the contributions of myelin and iron content to susceptibility was estimated.

\section{Conclusion}

We have developed a novel strategy to simultaneously acquire data for the reconstruction of five intrinsically co-registered 1-mm isotropic resolution multiparametric maps, with a scan time of 6 minutes using JVC-GRAPPA.

Keywords: Quantitative susceptibility mapping, myelin sensitive imaging, parallel imaging, multi-parametric imaging, MRI 


\section{Introduction}

Quantitative susceptibility mapping (QSM) has emerged as a promising technique for measuring magnetic susceptibility, which has been shown to correlate with paramagnetic substrates in the brain, including iron which is critical for cellular function. ${ }^{1,2}$ Unfortunately, deriving susceptibility from phase data requires solving an illconditioned linear system, which leads to image artifacts. To address this shortcoming, it becomes necessary to impose spatial regularization ${ }^{3-5}$ or acquire additional volumes at different head orientations (COSMOS), the latter which has been shown to generate higher quality susceptibility calculations. ${ }^{6}$

Myelin, which is comprised of a lipid and protein bilayer, forms sheaths around axons and facilitates neural conduction. Myelin content can be probed using $\mathrm{T}_{2^{-}}$ relaxometry ${ }^{7}$, quantitative magnetization transfer (qMT) ${ }^{8}$, macromolecular tissue volume $(\mathrm{MTV})^{9}$, and direct visualization of short transverse relaxation time component ${ }^{10}$. MTV estimates macromolecule content, and is highly correlated with qMT and $T_{2^{-}}$ relaxometry. ${ }^{9,11}$ Importantly, MTV offers a straightforward acquisition with high spatial resolution and signal-to-noise ratio $(\mathrm{SNR})^{12,13}$.

The information conferred through QSM and MTV could have important clinical applications in the management of neurological disorders that perturb myelin and iron content. $^{9,14-16}$ Although both are acquired using GRE sequences, they are traditionally obtained separately. Unfortunately, this can lead to lengthy scan times and image misregistration, complicating quantitative analyses. Herein, we exploit inherent redundancies between these two sequences by proposing a novel strategy to acquire whole-brain multi-orientation QSM and MTV simultaneously within the scan time of only one contrast. Additional quantitative parametric maps can be generated from the acquired data, including $T_{1}, R_{2}{ }^{*}$, and $P D$. Furthermore, we show that these multiparametric maps can be leveraged to produce myelin and iron-sensitive susceptibility maps.

To enhance clinical feasibility, we propose the use of Joint Virtual Coil generalized autocalibrating partially parallel acquisitions (JVC-GRAPPA). ${ }^{17}$ Parallel imaging techniques such as GRAPPA allow for reduced image acquisition times. ${ }^{18} 19$ The weighting coefficients (GRAPPA kernels) are derived from a fully-sampled 
autocalibration signal (ACS) region. Unfortunately, acceleration factors higher than $\mathrm{R}=3$ along one phase-encoding axis are generally avoided due to SNR degradation. ${ }^{20}$ To address this limitation, additional virtual coils (VC) can be generated using the complex conjugate symmetric k-space signals from the actual coils. The conjugate phase information provided by the VCs improves reconstruction and image quality. ${ }^{19,21,22}$

JVC-GRAPPA expands upon this by jointly reconstructing multiple echoes within the GRAPPA framework. In effect, a particular channel receives contributions from all image contrasts across all channels, while the phase information is converted into additional spatial encoding. ${ }^{19,21}$ We hypothesized that JVC-GRAPPA would yield image quality comparable to GRAPPA reconstructions while significantly reducing scan time.

The goals of our study were to 1) develop an acquisition scheme for collection of data suitable for reconstruction of QSM and MTV, reducing scan time to that of a single contrast, and 2) further reduce scan time using JVC-GRAPPA. If successful, our strategy could facilitate quantitative evaluation of neurological diseases.

\section{Methods}

MTV is acquired using a multi-flip angle (FA) 3D-GRE sequence with a relatively long repetition time (TR) and short echo time (TE). ${ }^{9}$ For COSMOS QSM reconstruction, a 3D-GRE sequence is also utilized to acquire data at three or more head orientations with a relatively long TE for improved phase contrast. ${ }^{23}$ Herein, we exploit the unused TR time in the MTV acquisition to collect additional late echoes for QSM processing. Additionally, we collect a different FA at each head orientation, allowing for simultaneous MTV and COSMOS reconstruction without scan time penalty.

To further accelerate the acquisition, we employ JVC-GRAPPA which jointly reconstructs multi-echo images by treating data from other echoes as extra coils, improving parallel imaging capability. ${ }^{17}$ The VC concept is applied to double the number of channels. The k-space signal received from a coil can be represented by the spatial spin-density distribution weighted by the spatial complex coil s'ensitivity. ${ }^{21}$ The symmetric complex-conjugate signal can be interpreted as the signal received by a virtual coil. While the magnitude sensitivity from the virtual coil is the same as the actual coil, the phase is different, providing additional encoding power. As each coil 
receives contributions from all coils and all echoes, for $N_{c}$ coils and $N_{e}$ echoes, we would train $\left(2 \times \mathrm{N}_{\mathrm{c}} \times \mathrm{N}_{\mathrm{e}}\right)^{2}$ kernels.

To address the problem that arises from the large number of kernels that need to be estimated (proportional to square of the number of channels), an iterative procedure is performed with an initial joint GRAPPA reconstruction. The entire k-space data estimated using the initial joint GRAPPA are then utilized in training successive JVCGRAPPA kernels ${ }^{19}$ Additionally, k-space sampling patterns of the individual echoes are shifted with respect to each other, providing complementary frequency information.

\subsection{Data Acquisition}

Three healthy adult volunteers were scanned on a 3T scanner (Siemens Skyra) with a 64-channel head coil in accordance with IRB-approved protocol. $^{24}$

Subject \#1: 3D multi-echo GRE sequence was acquired at three head orientations $\left(0^{\circ}, 7^{\circ}\right.$, and $13^{\circ}$ with respect to $B_{0}$; the same approximate orientations were also acquired for Subjects \#2-4) with the following parameters: 3 echoes, $\mathrm{TE}_{1}=5.73 \mathrm{~ms}$, echo spacing=9.14 ms, TR=31 ms, $F O V=192 \times 256 \times 160 \mathrm{~mm}^{3}$, and the vendor's $\mathrm{R}=2 \times 2$ GRAPPA. A different FA $\left(4^{\circ}, 10^{\circ}, 20^{\circ}\right)$ was applied at each head orientation. Rapid $\mathrm{B}_{1}+$ mapping calibration was acquired at each orientation using a 3D-FLASH sequence (acquisition time $=31$ seconds at each orientation). ${ }^{25}$ The total acquisition time was approximately $15 \mathrm{~min}$ and $17 \mathrm{~min}$ with $\mathrm{B} 1+$ mapping. Additional single orientation data was separately acquired.

Subject \#2: Fully sampled data were acquired at three head orientations (acquisition time $=60 \mathrm{~min} ; 62 \mathrm{~min}$ with $\mathrm{B} 1+)$, from which undersampling was retrospectively performed.

Subject \#3: Prospective undersampling was performed including $\mathrm{R}=3 \times 2$ GRAPPA (acquisition time $=9 \mathrm{~min} ; 11 \mathrm{~min}$ with $\mathrm{B} 1+$ ) and $\mathrm{R}=3 \times 3$ JVC-GRAPPA reconstruction (acquisition time $=6 \mathrm{~min} ; 8 \mathrm{~min}$ with $\mathrm{B} 1+$ ). The latter was enabled by introducing gradient blips to the sequence to produce k-space shifting at later echoes.

Subject \#4: To evaluate the reproducibility of multi-orientation MTV, scan-rescan was performed using the imaging same parameters as Subject \#1. This included two sets of multi-orientation and single orientation 3D multi-echo GRE data.

\subsection{Data Processing}


Subject \#1's data were reconstructed with the vendor's online GRAPPA. From subject \#2's fully-sampled data, undersampling was performed retrospectively along two phaseencoding axes by acceleration factors of $R=3 \times 2$ for GRAPPA and $R=3 \times 3$ for JVCGRAPPA. An ACS region of size $24 \times 24$ and a kernel size of $3 \times 3$ were used for both methods. During reconstruction, Tikhonov-regularization parameters were used for kernel calibration of $R=3 \times 2$ GRAPPA $\left(\lambda=10^{-8}\right)$ and $R=3 \times 3$ JVC-GRAPPA $\left(\lambda=10^{-7}\right)$ to optimize for root mean squared error (RMSE) relative to the fully-sampled ground truth. For subject \#3, in order to provide complementary k-space information, the sampling pattern was shifted by $(1,1)$ in the second echo, $(2,2)$ in the third echo, and $(3,3)$ in the fourth echo relative to the first echo.

For susceptibility, the raw phase data were processed using Laplacian-based phase unwrapping. ${ }^{26}$ Background phase removal was performed using V-SHARP in STI-Suite with a maximum kernel size of 25 voxels. The tissue phase images were then registered to the neutral orientation using FSL-FLIRT (https://fsl.fmrib.ox.ac.uk). COSMOS reconstruction was performed using an iterative least-squares formulation. ${ }^{14}$

For MTV, the $\mathrm{B}_{1}+$ map at each head orientation was co-registered to the corresponding GRE magnitude image using FSL-FLIRT. Calculation of the effective FA for $\mathrm{B} 1+$ bias-correction at each orientation was performed using the double-flip angle method. $^{12,27}$ The bias-corrected magnitude images were then skull-stripped and registered to the neutral orientation. $T_{1}$ and $M_{0}$ (product of the coil reception profile and proton density [PD]) maps were calculated using weighted-least-squares fitting. ${ }^{9}$ The reception profile was estimated using a method based the linear relationship between $\mathrm{PD}$ and $\mathrm{T} 1$ as previously described. ${ }^{28,29}$ PD maps were subsequently normalized to CSF-only voxels (WF), from which MTV was calculated.

$\mathrm{R}_{2}{ }^{*}$-mapping was performed using a least-squares mono-exponential fit of the multiecho magnitude data. The Isqnonneg function in Matlab (v.R2015b) was used to apply a nonnegative constraint to generate realistic values.

\subsection{Data Analysis}

To compare multi-orientation to single-orientation MTV in subject \#1, we coregistered the multi-orientation GRE acquisitions to the neutral frame using niftyreg (http://cmictig.cs.ucl.ac.uk/wiki/index.php/NiftyReg) after correcting for $\mathrm{B}_{1+}$ 
inhomogeneity. The co-registered images were compared to their single-orientation counterparts using RMSE. This was also performed for the MTV maps (multi- versus single-orientation). In addition, regions of interests (ROls) were manually placed by a board-certified neuroradiologist (F.Y., 8 years of experience) within selected brain regions using 3D Slicer (v.4.10.2, https://www.slicer.org/). ${ }^{23,30}$ Pearson correlation was used to measure the correlation between the single- versus multi-orientation MTV ROIs.

For subject \#2's retrospectively undersampled data, RMSE was calculated for $\mathrm{R}=3 \times 2$ GRAPPA and $\mathrm{R}=3 \times 3$ JVC-GRAPPA relative to fully sampled data. To assess similarity of prospectively undersampled JVC-GRAPPA to GRAPPA in subject \#3, RMSE was computed between the two sets of reconstructed images.

\subsection{Contribution of myelin and iron to susceptibility}

A potential application of the multiparametric maps is in separating the contributions of iron and myelin to susceptibility. We computed iron-induced and myelin-induced susceptibility maps based on the SEMI-TWInS method. ${ }^{31}$ SEMI-TWInS employs a three-compartment tissue model composed of iron (Fe), myelin (My), and extracellular matrix (M). Two key assumptions are made about the relationship between $R_{2}{ }^{*}$, bulk susceptibility $\chi$, iron concentration $C_{F e}$, and myelin concentration $C_{M y}$. The first states that $\mathrm{R}_{2}{ }^{*}$ and $\chi$ can be derived using linear combinations of $C_{F e}$ and $C_{M y}$, wherein

$$
\begin{aligned}
R_{2}^{*}\left(C_{F e}, C_{M y}\right) & \approx R_{2}^{*}+R_{2}^{*}+R_{2}^{*} \approx a_{1} \cdot C_{F e}+a_{2} \cdot C_{M y}+a_{3} \\
\chi\left(C_{F e}, C_{M y}\right) & \approx \chi_{F e}+\chi_{M y}+\chi_{M} \approx b_{1} \cdot C_{F e}+b_{2} \cdot C_{M y}+b_{3}
\end{aligned}
$$

The second assumption states that each coefficient $\left(a_{1}, a_{2}, a_{3}, b_{1}, b_{2}, b_{3}\right)$ maintains a constant value regardless of brain tissue type, and is unique to each individual. Of note, $C_{F e}$ and $C_{M y}$ represent spatially varying quantitative maps while $a_{1}, a_{2}, a_{3}, b_{1}, b_{2}, b_{3}$ are constants.

The six coefficients were computed by first determining $\mathrm{R}_{2}{ }^{*}, \chi, C_{F e}$, and $C_{M y}$ and subsequently solving Equations 1 and 2 using the NumPy linear least-squares solver. ROls were manually drawn in ten brain regions. Within the $\mathrm{ROIs}, \mathrm{R}_{2}{ }^{*}, \chi$, and $C_{M y}$ (MTV) were measured on a voxel-wise basis while $C_{F e}$ was taken from literature values. $^{32}$ The coefficients were computed by consolidating $\mathrm{R}_{2}{ }^{*}, \chi, C_{F e}$, and $C_{M y}$, and then least-squares solving Eqs. (1) and (2). 
was then computed in the brain by solving Equations 1 and 2 with respect to $R_{2}{ }^{*}$, , and the estimated coefficient values ( ). Iron-induced susceptibility and myelin-induced susceptibility maps were computed as and , respectively.

\section{Results}

\subsection{Single versus multi-orientation}

Previous studies used a single head orientation to estimate MTV, whereas we implemented a multiple head orientations approach. ${ }^{9,16}$ The multi-orientation $\mathrm{B}_{1+}$ corrected magnitude data had RMSEs of $4.8-5.8 \%$ relative to single orientation data (the same neutral orientation $\mathrm{FA}=20^{\circ}$ data was used for multi- and single-orientation). Multi-orientation MTV demonstrated an RMSE of $4.5 \%$ compared to single-orientation MTV (Figure 1), with the two maps being strongly correlated (Supporting Information Figures $\mathrm{S} 1$ and $\mathrm{S} 2)$. Additional parametric maps $\left(\mathrm{T}_{1}, \mathrm{PD}, \mathrm{R}_{2}{ }^{*}\right)$ were generated without added scan time (Figure 2).

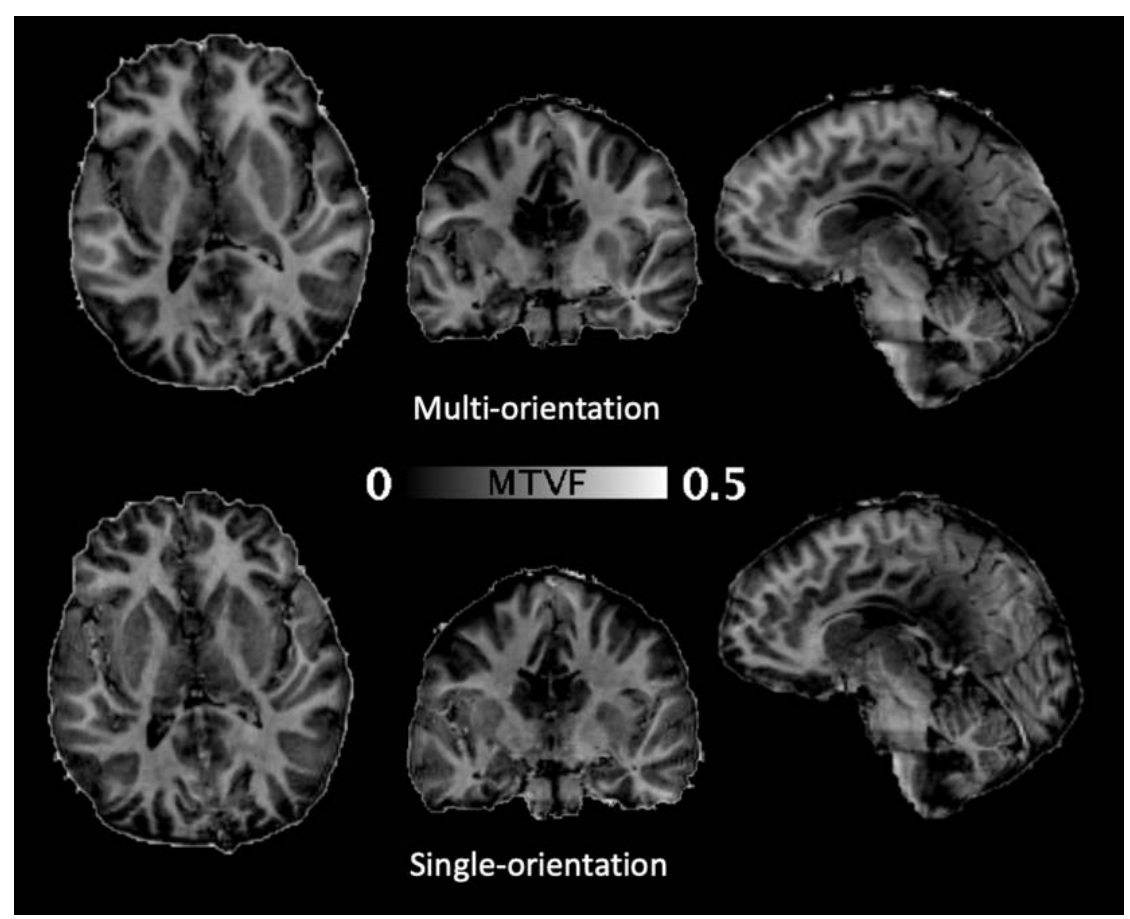

Figure 1: MTV maps acquired using single- (bottom row) and multiple-head orientations (bottom row) for Subject \#1, which demonstrate grossly similar results. 


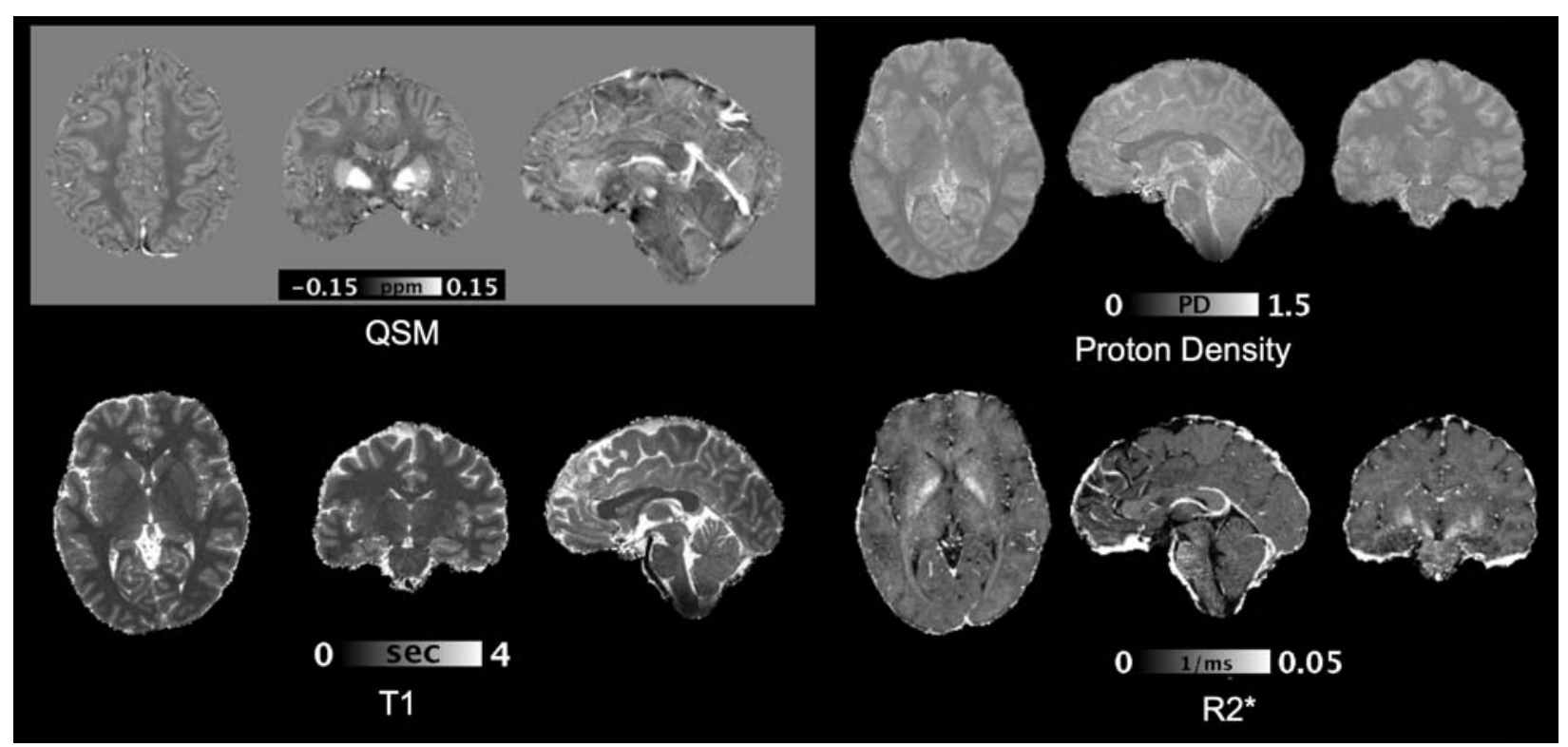

Figure 2: Representative multi-parametric maps ( $Q S M, R 2 *, T 1$, and $P D)$ generated from multi-orientation data for Subject \#2.

Scan-rescan data for Subject \#4 demonstrated an RMSE of 3\% between the initial and the repeat multi-orientation MTV maps, as compared to an RMSE of $3.2 \%$ between initial set of multi-orientation and single orientation MTV maps (Supporting Information Figure S3). The single orientation data demonstrated an RMSE of $1.8 \%$ between the initial and repeat single-orientation MTV data.

3.2. Fully sampled versus retrospectively undersampled data

For $\mathrm{FA}=4^{\circ}, \mathrm{R}=3 \times 2$ GRAPPA and $\mathrm{R}=3 \times 3$ JVC-GRAPPA yielded RMSEs of $3.1 \%$ and $3.5 \%$ with respect to $R=1$ data. For $F A=10^{\circ}$, GRAPPA and JVC-GRAPPA yielded RMSEs of $4.2 \%$ and $4.7 \%$, respectively. RMSEs for $F A=20^{\circ}$ were $5.3 \%$ and $5.7 \%$ for GRAPPA and JVC-GRAPPA, respectively. Comparing retrospectively undersampled MTV maps to R=1, GRAPPA and JVC-GRAPPA demonstrated RMSEs of $7.5 \%$ and 7.7\% (Supporting Information Figures S4 and S5).

\subsection{Prospectively acquired undersampled data}

Comparing JVC-GRAPPA magnitude images to GRAPPA, RMSEs for $F A=4^{\circ}, 10^{\circ}$, and $20^{\circ}$ were $3.1 \%, 5.1 \%$, and $6.5 \%$, respectively. Moreover, JVC-GRAPPA retained higher SNR, particularly for later echoes (Figure 3). 


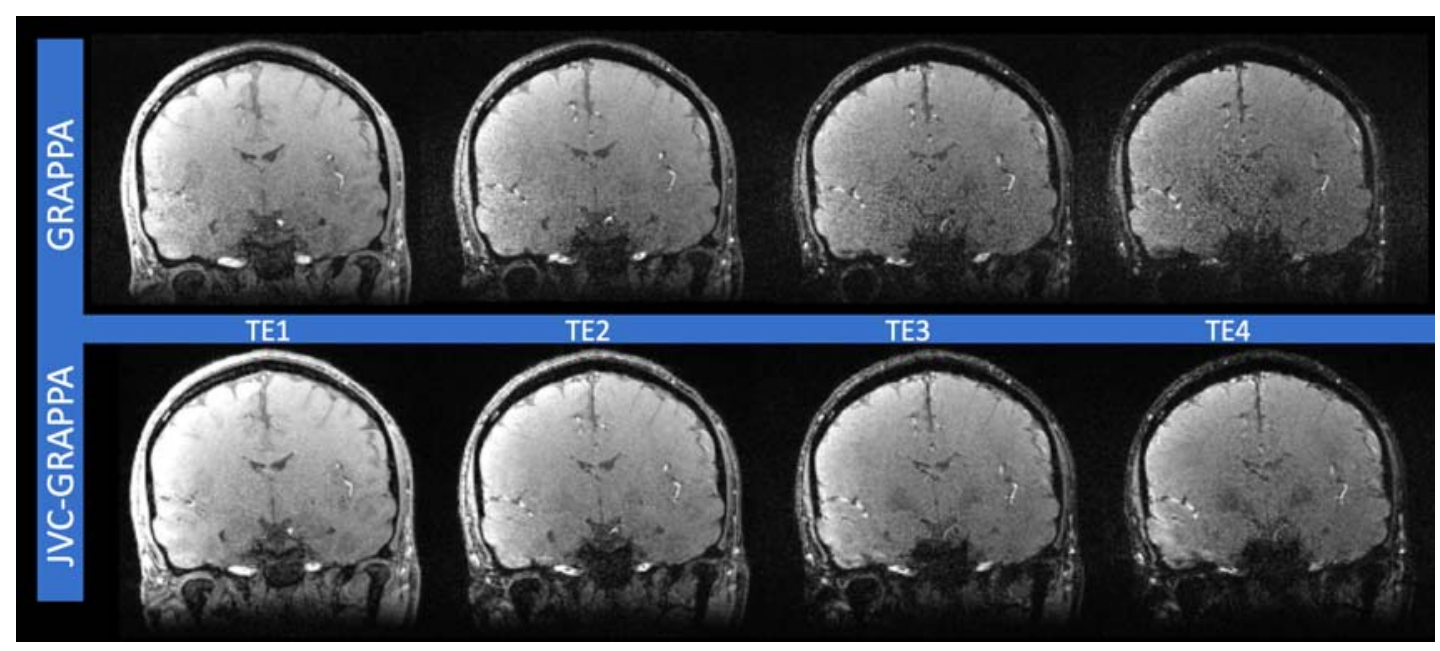

Figure 3: Prospectively under-sampled multi-echo GRE magnitude images obtained using $R=6$ GRAPPA (top row) and $R=9$ JVC GRAPPA (bottom row) from Subject \#3. JVC-GRAPPA reconstruction demonstrates higher SNR particularly for late echoes.

QSM demonstrated an RMSE of $1.4 \%$ between JVC-GRAPPA and GRAPPA, with no significant differences in susceptibility values $(p>0.1$; Supporting Information Figures $S 6$ and S7). Comparing JVC-GRAPPA and GRAPPA reconstruction-based MTV yielded an RMSE of $5.9 \%$ (Figure 4; Supporting Information Figures S8 and S9), with larger standard deviations observed with GRAPPA ( $p=0.002)$. We note that in the absence of ground truth, RMSEs computed between JVC-GRAPPA and GRAPPA aim to measure their similarity.

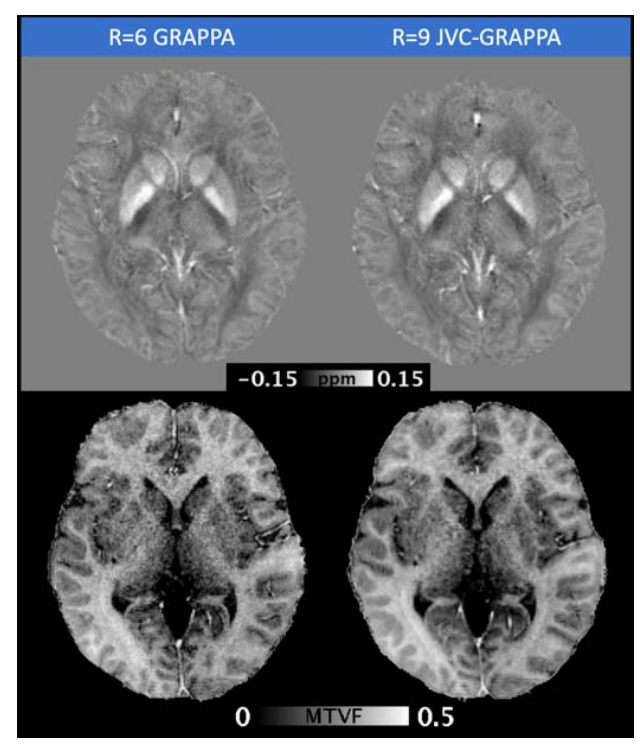

Figure 4: QSM (top) and MTV (bottom) maps generated from prospectively undersampled R=6 GRAPPA (left) and $R=9$ JVC-GRAPPA (right) reconstructions from Subject \#3. No significant differences can be observed with visual inspection. 


\subsection{Myelin and iron contributions to susceptibility}

Linear least-squares fitting yielded the following coefficients: $\quad=0.001$

$\mathrm{Hz}^{*} 100 \mathrm{mg} / \mathrm{mg} ; \quad=0.013 \mathrm{~Hz} / \mathrm{MTV} ; \quad=0.012 \mathrm{~Hz} ; \quad=0.009 \mathrm{ppm}{ }^{*} \mathrm{~g} / \mathrm{mg} ; \quad=-0.040$ $\mathrm{ppm} / \mathrm{MTV} ; \quad=-0.022 \mathrm{ppm}$. The map demonstrated significant differences from

(Figure 5). The hypointense signal within the map corresponds to negative susceptibility values, reflecting diamagnetic myelin. Image contrast was particularly striking between grey and white matter. Within deep gray nuclei, myelin-induced susceptibility contributions could be observed, although the iron-related contribution was predominant.

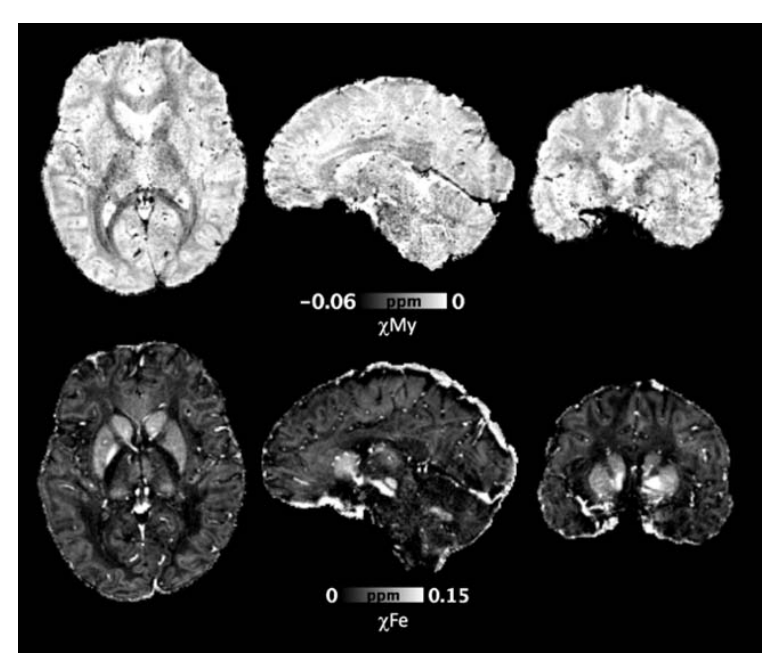

Figure 5: Representative myelin ( $\chi M y)$ and iron ( $\chi F e)$-induced susceptibility maps presented in axial, sagittal, and coronal projections. Diamagnetic signal is present within the white matter, but also the subcortical grey matter, consistent with the known distribution of myelin within the brain. Paramagnetic signal is most pronounced within the subcortical grey matter, but also seen within the cerebral cortex and to a lesser degree within the white matter.

\section{Discussion}

We propose an acquisition strategy that leverages inherent redundancies to obtain MTV and QSM within the same scan time as one parametric map, permitting concurrent interrogation of myelin content and magnetic susceptibility. We were also able to obtain three additional parametric maps $\left(\mathrm{PD}, \mathrm{T}_{1}, \mathrm{R}_{2}{ }^{*}\right)$ without scan time penalty. By applying JVC-GRAPPA reconstruction, we reduced the total acquisition time to 6 minutes.

\subsection{JVC-GRAPPA}

Our multi-echo acquisition supports the use of joint reconstruction, which was leveraged to decrease scan time, making the proposed method more suitable for clinical use. The 
shifted undersampling patterns of k-space across later echoes allowed for increased frequency coverage. In addition, VC concept was utilized to double the number of available channels to enhance spatial encoding. While there was noise amplification resulting from the intrinsic SNR penalty associated with parallel imaging, the resulting images had improved SNR compared to the slower GRAPPA.

One potential limitation is that because the method stacks data from all image contrasts along the coil axis, larger amounts of calibration data are required. The shifted k-space sampling further contributes to this as the staggered JVC kernels span a larger k-space extent, expanding the ACS region. ${ }^{17}$ To alleviate this, we used an initial joint GRAPPA reconstruction to provide an estimate of the entire k-space matrix, which was used for calibrating the following JVC steps. Compared to other highly-accelerated methods, advantages of JVC-GRAPPA include its relative ease in processing and lower computational demands, which facilitate potential online reconstruction. ${ }^{17}$

\section{2. $\quad$ MTV}

Previous studies have acquired multi-FA GRE images in a single head orientation. ${ }^{9}$ In this study, after performing $B_{1}+$ inhomogeneity correction for each orientation, the RMSE between the magnitude images was minimal. ${ }^{33}$ Although GRE magnitude images are influenced by orientation of tissues relative to $B_{0}$, we found that the resultant multi- and single-orientation MTV maps yielded similar results. ${ }^{30}$

The GRE images demonstrated minimal differences between R=9 JVC-GRAPPA and $R=6$ GRAPPA reconstructions. MTV demonstrated a slightly greater difference between acceleration methods, which likely reflects additional noise amplification as a result of the intervening processing steps.

\section{3. $\underline{\mathrm{QSM}}$}

COSMOS reconstruction has been shown to provide higher quality susceptibility estimates than regularized single orientation methods. ${ }^{6,14}$ Generally, single orientation reconstructions can suffer from streaking artifacts as well as over-smoothing. However, in order to achieve optimal imaging using COSMOS, sampling at a minimum of three head orientations is required. ${ }^{23}$ Significant deviations, either through fewer than three 
acquisitions or reducing the angle between sampling orientations, can result in streaking artifacts.

We acknowledge that even with reduced scan time, there are patients for whom adequately rotating the head may prove challenging. In these circumstances, the proposed method can be acquired using a single head orientation, with subsequent application of a QSM regularization method. ${ }^{6,34}$ Although the estimation within anisotropic tissues could vary depending on head orientation due to susceptibility anisotropy ${ }^{35}$, isotropic structures or those with greater intrinsic susceptibility (deep grey nuclei) should appear similar to COSMOS. ${ }^{30,34}$

\subsection{Additional contrasts}

$\mathrm{R}_{2}{ }^{*}, \mathrm{~T}_{1}$, and PD maps were also generated from the acquired data without additional scan time cost. $R_{2}{ }^{*}$ is influenced by myelin and iron content. ${ }^{36}$ While $R_{2}{ }^{*}$ can be calculated from a single orientation, combining all three yielded higher SNR. $\mathrm{T}_{1}$ mapping has potential applications in the evaluation of hepatic encephalopathy ${ }^{37}$ and multi-system atrophy ${ }^{38}$. Recently, Filo et al. used a combination of parametric measures, including $\mathrm{T} 1$ (R1) and MTV, to reveal region-specific molecular changes associated with aging. ${ }^{39}$ A similar strategy could be applied to detect pathologic deviations from normal aging that occur in neurologic diseases.

We showed that by leveraging the intrinsically co-registered multi-parametric maps $\left(\mathrm{R}_{2}{ }^{*}\right.$, susceptibility, MTV), high-spatial-resolution parametric maps of iron and myelininduced susceptibility can be generated within the same anatomic space. These maps are relevant because although studies have supported using QSM as a brain iron marker, myelin also affects susceptibility particularly within white matter ${ }^{40}$. It is important to acknowledge that MTV is not entirely specific for myelin and is affected by other diamagnetic macromolecules ${ }^{9}$. Likewise, in certain brain regions, other non-iron paramagnetic substrates could influence susceptibility. Nonetheless, the ability to approximate the voxel-wise contributions of myelin and iron to susceptibility enhances the clinical utility of magnetic susceptibility imaging. ${ }^{41}$ Potential applications include demyelinating diseases, traumatic brain injury, and neurodegenerative diseases. 


\subsection{Limitations}

Joint parallel imaging is associated with increased reconstruction time compared to GRAPPA. $^{17}$ Applying the JVC kernels using a less demanding element-wise multiplication in image space (rather than convolution in k-space) could help address this. To delineate susceptibility contributions from iron, we utilized literature reference values for iron concentration for calibration. We also note that paramagnetic and diamagnetic substrates other than myelin and iron may contribute to the observed susceptibility. Future plans include exploring alternative calibration schemes that may allow for more robust estimations in neuropathology. A current limitation is our small study size, restricting generalizability of the results. To realize the potential of our quantitative imaging approach, it will be necessary to image additional subjects and across different sites. ${ }^{42}$

\section{Conclusion}

In this study, we proposed a strategy for simultaneously acquiring QSM and MTV by exploiting their inherent redundancies to reduce scan time. We also generated multiple quantitative parametric maps, including $\mathrm{T}_{1}, \mathrm{R}_{2}{ }^{*}, \chi_{M y}, \chi_{F e}$, and $\mathrm{PD}$ without scan time penalty. Clinical feasibility was enhanced by further accelerating the acquisition by 9fold using JVC-GRAPPA while preserving image quality. This protocol has potential applications in the quantitative evaluation of neurological diseases.

\section{References}

1 Langkammer, C. et al. Quantitative susceptibility mapping (QSM) as a means to measure brain iron? A post mortem validation study. Neurolmage 62, 1593-1599, doi:10.1016/j.neuroimage.2012.05.049 (2012).

2 Stephenson, E., Nathoo, N., Mahjoub, Y., Dunn, J. F. \& Yong, V. W. Iron in multiple sclerosis: roles in neurodegeneration and repair. Nature Reviews Neurology 10, 459-468, doi:10.1038/nrneurol.2014.118 (2014).

3 De Rochefort, L. et al. Quantitative susceptibility map reconstruction from MR phase data using bayesian regularization: Validation and application to brain imaging. Magnetic Resonance in Medicine 63, 194-206, doi:10.1002/mrm.22187 (2010). 
$4 \quad \mathrm{Li}, \mathrm{W} ., \mathrm{Wu}, \mathrm{B}$. \& Liu, C. Quantitative susceptibility mapping of human brain reflects spatial variation in tissue composition. Neurolmage 55, 1645-1656, doi:10.1016/j.neuroimage.2010.11.088 (2011).

5 Schweser, F., Sommer, K., Deistung, A. \& Reichenbach, J. R. Quantitative susceptibility mapping for investigating subtle susceptibility variations in the human brain. Neurolmage 62, 2083-2100, doi:10.1016/j.neuroimage.2012.05.067 (2012).

6 Liu, T. et al. Morphology enabled dipole inversion (MEDI) from a single-angle acquisition: Comparison with COSMOS in human brain imaging. Magnetic Resonance in Medicine 66, 777-783, doi:10.1002/mrm.22816 (2011).

7 Mackay, A. et al. In vivo visualization of myelin water in brain by magnetic resonance. Magnetic Resonance in Medicine 31, 673-677 (1994).

8 Henkelman, R. M. et al. Quantitative interpretation of magnetization transfer. Magnetic Resonance in Medicine 29, 759-766, doi:10.1002/mrm.1910290607 (1993).

9 Mezer, A. et al. Quantifying the local tissue volume and composition in individual brains with magnetic resonance imaging. Nature Medicine 19, 1667-1672, doi:10.1038/nm.3390 (2013).

10 Oh, S.-H. et al. Direct visualization of short transverse relaxation time component (ViSTa). Neurolmage 83, 485-492, doi:10.1016/j.neuroimage.2013.06.047 (2013).

11 West, K. L. et al. Myelin volume fraction imaging with MRI. Neurolmage, doi:10.1016/j.neuroimage.2016.12.067 (2016).

12 Duval, T. et al. g-Ratio weighted imaging of the human spinal cord in vivo. Neurolmage 145, 11-23, doi:10.1016/j.neuroimage.2016.09.018 (2017).

13 Berman, S., West, K. L., Does, M. D., Yeatman, J. D. \& Mezer, A. A. Evaluating g-ratio weighted changes in the corpus callosum as a function of age and sex. Neurolmage, doi:10.1016/j.neuroimage.2017.06.076 (2017).

14 Bilgic, B. et al. Rapid multi-orientation quantitative susceptibility mapping. Neurolmage 125, 1131-1141, doi:10.1016/j.neuroimage.2015.08.015 (2016).

15 Guo, A. C., Jewells, V. L. \& Provenzale, J. M. Analysis of Normal-Appearing White Matter in Multiple Sclerosis: Comparison of Diffusion Tensor MR Imaging and Magnetization Transfer Imaging. American Journal of Neuroradiology 22, 1893-1900 (2001).

$16 \mathrm{Yu}, \mathrm{F}$. et al. Imaging G-Ratio in Multiple Sclerosis Using High-Gradient Diffusion MRI and Macromolecular Tissue Volume. AJNR Am J Neuroradiol 40, 1871-1877, doi:10.3174/ajnr.A6283 (2019).

17 Bilgic, B. et al. Improving parallel imaging by jointly reconstructing multi-contrast data. Magnetic Resonance in Medicine, doi:10.1002/mrm.27076 (2018).

18 Griswold, M. A. et al. Generalized autocalibrating partially parallel acquisitions (GRAPPA). Magnetic Resonance in Medicine 47, 1202-1210, doi:10.1002/mrm.10171 (2002).

19 Blaimer, M. et al. Comparison of phase-constrained parallel MRI approaches: Analogies and differences. Magnetic Resonance in Medicine 75, 1086-1099, doi:10.1002/mrm.25685 (2016).

20 Pruessmann, K. P., Weiger, M., Scheidegger, M. B. \& Boesiger, P. SENSE: sensitivity encoding for fast MRI. Magn Reson Med 42, 952-962 (1999). 
21 Blaimer, M. et al. Virtual coil concept for improved parallel MRI employing conjugate symmetric signals. Magnetic Resonance in Medicine 61, 93-102, doi:10.1002/mrm.21652 (2009).

22 Blaimer, M., Jakob, P. M. \& Breuer, F. A. Regularization method for phase-constrained parallel MRI. Magnetic Resonance in Medicine 72, 166-171, doi:10.1002/mrm.24896 (2014).

23 Liu, T., Spincemaille, P., de Rochefort, L., Kressler, B. \& Wang, Y. Calculation of susceptibility through multiple orientation sampling (COSMOS): a method for conditioning the inverse problem from measured magnetic field map to susceptibility source image in MRI. Magnetic Resonance in Medicine 61, 196-204, doi:10.1002/mrm.21828 (2009).

24 Keil, B. et al. A 64-channel 3T array coil for accelerated brain MRI. Magnetic Resonance in Medicine 70, 248-258, doi:10.1002/mrm.24427 (2013).

25 Yarnykh, V. L. Actual flip-angle imaging in the pulsed steady state: A method for rapid three-dimensional mapping of the transmitted radiofrequency field. Magnetic Resonance in Medicine 57, 192-200, doi:10.1002/mrm.21120 (2007).

26 Li, W., Avram, A. V., Wu, B., Xiao, X. \& Liu, C. Integrated Laplacian-based phase unwrapping and background phase removal for quantitative susceptibility mapping. NMR in Biomedicine 27, 219-227, doi:10.1002/nbm.3056 (2014).

27 Stollberger, R. \& Wach, P. Imaging of the active B1 field in vivo. Magn Reson Med 35, 246-251, doi:10.1002/mrm.1910350217 (1996).

28 Volz, S., Nöth, U. \& Deichmann, R. Correction of systematic errors in quantitative proton density mapping. Magn Reson Med 68, 74-85, doi:10.1002/mrm.23206 (2012).

29 Mezer, A., Rokem, A., Berman, S., Hastie, T. \& Wandell, B. A. Evaluating quantitative proton-density-mapping methods. Hum Brain Mapp 37, 3623-3635, doi:10.1002/hbm.23264 (2016).

$30 \mathrm{Li}$, J. et al. Reducing the object orientation dependence of susceptibility effects in gradient echo MRI through quantitative susceptibility mapping. Magn Reson Med 68, 1563-1569, doi:10.1002/mrm.24135 (2012).

31 Schweser, F., Deistung, A., Lehr, B. W., Sommer, K. \& Reichenbach, J. R. SEMI-TWInS: Simultaneous Extraction of Myelin and Iron using a T2*-Weighted Imaging Sequence. 1.

32 Hallgren, B. \& Sourander, P. The effect of age on the non-haemin iron in the human brain. 3, 41-51 (1958).

33 Fram, E. K. et al. Rapid calculation of T1 using variable flip angle gradient refocused imaging. Magn Reson Imaging 5, 201-208 (1987).

$34 \mathrm{Li}, \mathrm{W}$. et al. A method for estimating and removing streaking artifacts in quantitative susceptibility mapping. Neurolmage 108, 111-122, doi:10.1016/j.neuroimage.2014.12.043 (2015).

35 Li, W., Wu, B., Avram, A. V. \& Liu, C. Magnetic Susceptibility Anisotropy of Human Brain in vivo and its Molecular Underpinnings. Neuroimage 59, 2088-2097, doi:10.1016/j.neuroimage.2011.10.038 (2012).

36 Zhang, Y. et al. Quantitative Susceptibility Mapping and R2* Measured Changes during White Matter Lesion Development in Multiple Sclerosis: Myelin Breakdown, Myelin 
Debris Degradation and Removal, and Iron Accumulation. American Journal of Neuroradiology 37, 1629-1635, doi:10.3174/ajnr.A4825 (2016).

37 Shah, N. J. et al. Quantitative T1 mapping of hepatic encephalopathy using magnetic resonance imaging. Hepatology 38, 1219-1226, doi:10.1053/jhep.2003.50477 (2003).

38 Vymazal, J. et al. T1 and T2 in the brain of healthy subjects, patients with Parkinson disease, and patients with multiple system atrophy: relation to iron content. Radiology 211, 489-495, doi:10.1148/radiology.211.2.r99ma53489 (1999).

39 Filo, S. et al. Disentangling molecular alterations from water-content changes in the aging human brain using quantitative MRI. Nature Communications 10, doi:10.1038/s41467-019-11319-1 (2019).

40 Duyn, J. H. \& Schenck, J. Contributions to magnetic susceptibility of brain tissue. NMR in Biomedicine 30, doi:10.1002/nbm.3546 (2016).

$41 \mathrm{Yu}, \mathrm{F}$. F. et al. Characterization of normal-appearing white matter in multiple sclerosis using quantitative susceptibility mapping in conjunction with diffusion tensor imaging. Neuroradiology, doi:10.1007/s00234-018-2137-7 (2018).

42 Leutritz, T. et al. Multiparameter mapping of relaxation (R1, R2*), proton density and magnetization transfer saturation at $3 \mathrm{~T}$ : A multicenter dual-vendor reproducibility and repeatability study. Hum Brain Mapp 41, 4232-4247, doi:10.1002/hbm. 25122 (2020). 


\section{Figure Captions}

Figure 1: MTV maps acquired using single- (bottom row) and multiple-head orientations (bottom row) for Subject \#1 presented in axial, coronal, and sagittal projections, which demonstrate similar results.

Figure 2: Representative multi-parametric maps (QSM, R2* $T 1$, and PD) generated from multiorientation data for Subject \#2.

Figure 3: Prospectively under-sampled multi-echo GRE magnitude images obtained using $R=6$ GRAPPA (top row) and R=9 JVC GRAPPA (bottom row) from Subject \#3. JVC-GRAPPA reconstruction demonstrates higher SNR particularly for late echoes.

Figure 4: QSM (top) and MTV (bottom) maps generated from prospectively undersampled $\mathrm{R}=6$ GRAPPA (left) and R=9 JVC-GRAPPA (right) reconstructions from Subject \#3. No significant differences can be observed with visual inspection.

Figure 5: Representative myelin ( $\mathrm{XMy}$ ) and iron ( $\mathrm{XFe}$ )-induced susceptibility maps presented in axial, sagittal, and coronal projections. Diamagnetic signal is present within the white matter, but also the subcortical grey matter, consistent with the known distribution of myelin within the brain. Paramagnetic signal is most pronounced within the subcortical grey matter, but also seen within the cerebral cortex and to a lesser degree within the white matter.

Supporting Information Figure S1: Selected grey and white matter regions in the brain (Subject $\# 1$ ) with their corresponding values on single and multi-orientation MTV $(\square=0.965, p<0.0001)$.

Supporting Information Figure S2: 2D histogram of voxel-wise correlation over the whole brain between single and multi-orientation MTV for Subject \#1.

Supporting Information Figure S3: Scan-Rescan axial MTV images from Subject \#4 (from left to right: initial multi-orientation MTV, repeat multi-orientation MTV, and initial single-orientation MTV).

Supporting Information Figure S4: 2D histogram of voxel-wise correlation over the whole brain between fully sampled $R=1$ and retrospectively undersampled $R=6$ GRAPPA MTV for Subject \#2.

Supporting Information Figure S5: 2D histogram of voxel-wise correlation over the whole brain between fully sampled $R=1$ and retrospectively undersampled $R=9$ JVC-GRAPPA MTV for Subject \#2.

Supporting Information Figure S6: Selected grey and white matter regions with their corresponding values on $\mathrm{R}=6$ GRAPPA and $\mathrm{R}=9$ JVC-GRAPPA for QSM, respectively.

Supporting Information Figure S7: 2D histogram of voxel-wise correlation over the whole brain between prospectively undersampled $\mathrm{R}=6$ GRAPPA and $\mathrm{R}=9 \mathrm{JVC}$-GRAPPA QSM for Subject \#3. 
Supporting Information Figure S8: Selected grey and white matter regions with their corresponding values on $\mathrm{R}=6$ GRAPPA and $\mathrm{R}=9$ JVC-GRAPPA for MTV, respectively.

Supporting Information Figure S9: 2D histogram of voxel-wise correlation over the whole brain between prospectively undersampled $\mathrm{R}=6$ GRAPPA and $\mathrm{R}=9$ JVC-GRAPPA MTV for Subject \#3. 